\title{
A new method for accurately localizing and resecting pulmonary nodules
}

\author{
Gongming Wang ${ }^{1 \#}$, Yongyong Lin ${ }^{1 \#}$, Lie Zheng ${ }^{1}$, Yin Liang ${ }^{1}$, Lei Zhao ${ }^{1}$, Yinsheng Wen ${ }^{1}$, Rusi Zhang ${ }^{1}$, \\ Zirui Huang ${ }^{1}$, Longjun Yang', Dechang Zhao', Samy Lachkar ${ }^{2}$, Jean Marc Baste ${ }^{3}$, Naofumi Shinagawa \\ Calvin S. H. $\mathrm{Ng}^{5}$, Masaaki Sato ${ }^{6}$, Min P. Kim ${ }^{7}$, Lanjun $\mathrm{Zhang}^{1}$ \\ ${ }^{1}$ Department of Thoracic Surgery, Sun Yat-sen University Cancer Center, Guangzhou, China; ${ }^{2}$ Department of Pulmonology, Thoracic Oncology \\ and Respiratory Intensive Care \& CIC- CRB 1404, Rouen University Hospital, Rouen, France; ${ }^{3}$ Department of Thoracic Surgery, Rouen University \\ Hospital, Rouen, France; ${ }^{4}$ First Department of Medicine, Hokkaido University School of Medicine, Sapporo, Japan; ${ }^{5}$ Division of Cardiothoracic \\ Surgery, Department of Surgery, Prince of Wales Hospital, the Chinese University of Hong Kong, Hong Kong, China; ${ }^{6}$ Department of Thoracic \\ Surgery, The University of Tokyo Graduate School of Medicine, Tokyo, Japan; ${ }^{7}$ Division of Thoracic Surgery, Department of Surgery, Houston \\ Methodist Hospital, Houston, TX, USA \\ \#These authors contributed equally to this work. \\ Correspondence to: Lanjun Zhang. Department of Thoracic Surgery, Sun Yat-sen University Cancer Center, No. 651, Dongfeng East Road, \\ Guangdong 510030, China. Email: Zhanglj@sysucc.org.cn.
}

\begin{abstract}
With the use of low-dose CT for early screening of lung cancer, more and more early lung cancers are found. At the same time, patients with small lung nodules have also increased, it is a great challenge for surgeons to resect pulmonary nodules with small volume, deep position and no solid components under video-assisted thoracoscopic surgery. Many studies have reported preoperative and intraoperative methods for localizing lung nodules before minimally invasive resection. Methods for preoperative localization include CT-guided hook-wire positioning, coil positioning, or dye injection and radionuclide location Methods for intraoperative localization include intraoperative ultrasound localization and tactile pressure-sensing localization. After the localization of pulmonary nodules under the guidance of CT patients need to restrict their activities; otherwise, it is easy for the nodules to move, causing the operation to fail, and may also cause complications such as pneumothorax, puncture site pain, and pulmonary parenchymal bleeding. In the past, we injected melamine dye under the guidance of electromagnetic navigation bronchoscope to locate lung nodules. The purpose of this case is introducing a new method for accurately localizing and resecting pulmonary nodules by injecting indocyanine green (ICG) under the guidance of electromagnetic navigation bronchoscope and the resection of small pulmonary nodules under the fluoroscope.
\end{abstract}

Keywords: Pulmonary nodules; indocyanine green (ICG); electromagnetic navigation bronchoscope; fluoroscope

Submitted Jun 01, 2020. Accepted for publication Aug 11, 2020.

doi: 10.21037/jtd-20-2089

View this article at: http://dx.doi.org/10.21037/jtd-20-2089

\section{Introduction}

Lung cancer still has the highest incidence and mortality of malignant tumors in the world (1). According to China cancer statistics in 2015, the incidence and mortality of lung cancer in China rank first (2). The reasons for this are mainly that diagnosis occurs when the patients are already in the late stage of lung cancer, and the treatment effect is thus poor. With low-dose computed tomography (CT) being used in the early screening of lung cancer in recent years, an increasing number of patients with pulmonary nodules are being encountered. However, it is still difficult to determine if the nodules are benign or malignant. There are still many high-risk patients who are recommended for 
surgery according to their clinical imaging features, like burr, vascular and bronchial signs, and pleural traction. However, for ground-glass opacity (GGO) lesions, there is no solid component, so it is difficult for the operator to identify the location of the tumor through finger touch aloe. This is highly challenging for surgeons, especially younger ones. Previous researches on the localization methods of ground glass nodules in lung mainly include CT-guided Hook-wire positioning, CT-guided percutaneous puncture coil positioning, CT-guided percutaneous methylene blue staining, ICG or contrast agent positioning. Besides, the melocyanine injection guided by electromagnetic Navigation bronchoscopy is also a new way to localizate the lung nodules. However, dye diffusion and implant displacement (mainly occurring during patient transfer to Operation room) can cause localization failure. In addition, the risks of CT-guided transthoracic positioning surgery include pleural-related complications, such as pleural pain, pneumothorax, hemothorax, and embolism. We here report the use of electromagnetic navigation to guide the injection of a fluorescent agent to help surgeons more accurately complete the intraoperative localization of pulmonary pure model glass nodules. We present the following article in accordance with the CARE reporting checklist (available at http://dx.doi.org/10.21037/jtd-20-2089).

\section{Case presentation}

A 47-year-old female patient came to our hospital after a physical examination discovered nodules in her left upper lung. The chest CT showed that the left upper pulmonary nodule was about $7 \mathrm{~mm} \times 9 \mathrm{~mm}$ in size. There was no cough, expectoration, hemoptysis, dizziness, headache, chest tightness, asthma, bone pain, or other special symptoms. Prior to being admitted to our center, the patient received cephalosporin anti-inflammatory treatment for lung infection for 2 weeks, and the chest CT scan reexamination showed no changes after 3 months had occurred. She then came to our hospital for further treatment.

Her past medical history was as follows: (I) ovarian cyst operation in 2002; (II) ectopic pregnancy operation in 2006; (III) no blood transfusion or donation history; (IV) previous history of hepatitis b for 20 years; (V) no history of hypertension, diabetes, coronary heart disease, or other diseases.

Her personal history was as follows: no smoking or drinking history, and no history living in unhygienic or epidemic areas. As for her family cancer history, her mother had a history of breast cancer and died.

The patient's menstrual history was as follows: menarche at 13 years old, 5-day cycle, 30 days menstrual period; last menstruation in December 17; she experience regular, moderate menstruation with no dysmenorrhea. She had no food or drug allergies.

Physical examination revealed symmetry of the thoracic cage, no varicose veins in the chest wall, symmetry of the tactile sensation and speech fibrillation in both lungs, clear cause of percussion, clear breath sound in both lungs, and no dry and wet rales. The heart rhythm was homogeneous, no pathological murmur was heard in each valve. The laboratory examination results are shown in Table 1. From the table, we can see that the indicators of blood sampling examination of the patient after admission are basically normal. In addition, examinations for HIV (Human Immunodeficiency Virus) antibody, syphilis antibody, tuberculosis antibody, hepatitis 4, and two halves of hepatitis $\mathrm{b}$ were all negative. Chest and abdomen enhanced CT showed $9 \mathrm{~mm} \times 7 \mathrm{~mm}$ sized nodules in the posterior segment of the upper lobe of the left lung, with a clear boundary and uneven density. No abnormalities were found in craniocerebral magnetic resonance imaging (MRI), tracheoscopy, or cardiopulmonary function (Figures 1-3).

\section{Clinical diagnosis: lung cancer}

Therapeutic procedure: According to the preoperative chest CT results of the patient and the $3 \mathrm{D}$ reconstruction technology, the location of pulmonary nodule, the shape of the pulmonary vessels and trachea, and whether there was any variation were determined (Figure 4). Using "superDimension" software (Shanghai Medtronic, China), the axial, coronal, and sagittal views of the CT scan of the patient are used to build a 3D airway tree and plan the navigation path to the nodule. A single-lumen tracheal catheter was used to induce general endotracheal anesthesia in the operating room. Then locate each nodule on the navigation console, including the positionable electromagnetic catheter and the extended working catheter. During real-time navigation, the distance between the electromagnetic catheter and the nodules can be measured. After locating the lesion, adjust the position of the electromagnetic catheter with the nearest pleural surface to be greater than $1 \mathrm{~cm}$ to avoid perforation, then remove the electromagnetic catheter and inject icg into the lesion through the extended working catheter. After removing the extended working Catheter, remove the 
Table 1 Laboratory examination results (pre-op)

\begin{tabular}{|c|c|c|c|}
\hline Item & Results & $\begin{array}{c}\text { Normal/ } \\
\text { abnormal }\end{array}$ & Reference range \\
\hline \multicolumn{4}{|l|}{ Tumor marker } \\
\hline CyFRA21-1 (ng/mL) & 3.19 & - & $0.10-3.30$ \\
\hline CEA (ng/mL) & 0.795 & - & $0.00-5.00$ \\
\hline NSE (ng/mL) & 12.74 & - & $0.00-15.20$ \\
\hline \multicolumn{4}{|l|}{ Blood routine } \\
\hline WBC $\left(\times 10^{9} / \mathrm{L}\right)$ & 7.91 & - & $3.5-9.5$ \\
\hline HGB (g/L) & 144.0 & - & $115.0-150.0$ \\
\hline PLT (×10 9 /L) & 263 & - & $100.0-350.0$ \\
\hline \multicolumn{4}{|l|}{ Liver function test } \\
\hline ALT (U/L) & 7.6 & - & $7-40$ \\
\hline AST (U/L) & 18.1 & - & $13-35$ \\
\hline AS/AL & 2.38 & - & $0-3$ \\
\hline CHE (U/L) & 8,806 & - & $5,320-12,920$ \\
\hline ALP (U/L) & 45.8 & - & $35-100$ \\
\hline GGT (U/L) & 12.6 & - & $7-45$ \\
\hline LDH (U/L) & 166.2 & - & $120-250$ \\
\hline ALB (g/L) & 38.2 & - & $40-55$ \\
\hline GLOB (g/L) & 26.75 & - & $20-40$ \\
\hline GLU (mmol/L) & 6.96 & $\uparrow$ & $3.9-6.1$ \\
\hline CK (U/L) & 337 & $\uparrow$ & $40-200$ \\
\hline CRP (mg/L) & 166.27 & $\uparrow$ & $0-3$ \\
\hline
\end{tabular}

CyFRA21-1, cytokeratin-19-fragment; CEA, carcinoembryonic antigen; NSE, neuronspecific enolase; WBC, white blood cell count; HGB, hemoglobin; PLT, platelet count; ALT, alanine aminotransferase; AST, aspartate aminotransferase; CHE, cholinesterase; ALP, alkaline phosphatase; GGT, gamma glutamine transferase; LDH, lactate dehydrogenase; ALB, albumin; GLOB, globulin; GLU, glucose in the blood; CK, creatine kinase; CRP, C-reactive protein.

patient's airway secretions by bronchoscopy. and the left upper lung tip posterior segment resection was performed under thoracoscope on Jan. 18, 2019 (Figures 5-7).

Surgery approach: Intraoperative frozen examination revealed that the shape of the left upper lung nodule conformed to the adenocarcinoma, so it was necessary to distinguish the adenocarcinoma in situ from the invasive adenocarcinoma, so the $5^{\text {th }}, 7^{\text {th }}, 10^{\text {th }}$, and $12^{\text {th }}$ groups of lymph nodes were biopsied. The operation process was
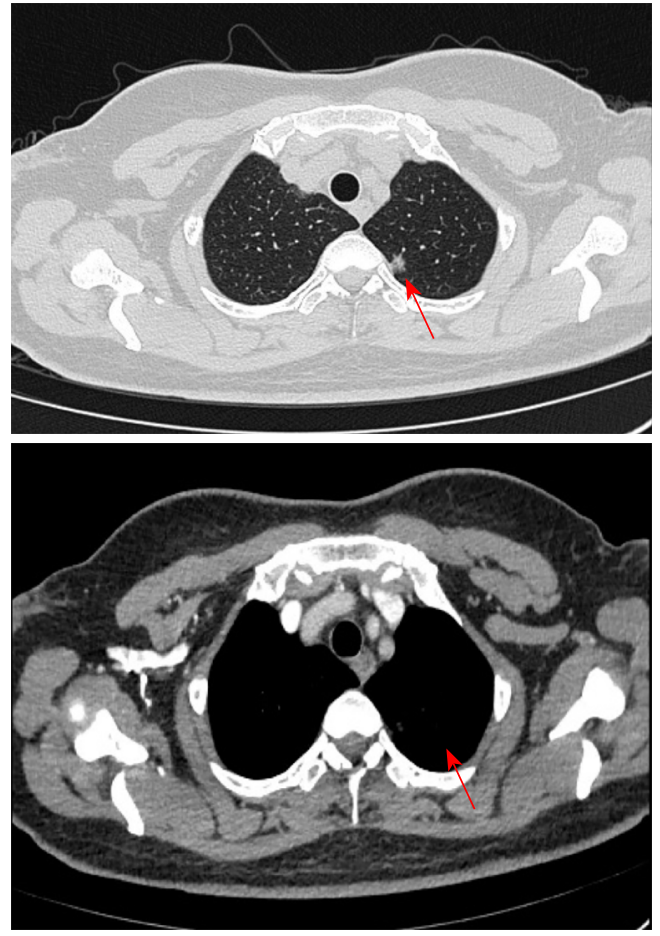

Figure 1 Chest and abdomen enhanced CT showing $9 \mathrm{~mm} \times$ $7 \mathrm{~mm}$ nodules found in the posterior segment of the upper lobe of the left lung, with a clear boundary and uneven density (as shown by the arrow).

smooth, the bleeding was $100 \mathrm{~mL}$ during the operation, the patients were given anti-inflammatory and symptomatic support treatment after the operation, and the patients were discharged smoothly.

Postoperative pathological report: After paraffin freezing, focal epithelial dysplasia was seen in the left upper lung tissue, and most of them adhered to the growth. The alveolar septum of the small focus was widened with fibrous connective tissue hyperplasia. Among them, small glandular duct-like structures were found. The lesions were consistent with adenocarcinoma in situ,

(I) One lymph node in the fifth group: no cancer.

(II) One lymph node in the seventh group: no cancer.

(III) Three lymph node in the tenth group: no cancer.

(IV) Two lymph node in the twelfth group: no cancer.

Postoperative stage: left upper lung adenocarcinoma in situ.

Gene test results are as below: (epidermal growth factor receptor, EGFR) (-), (KRAS proto-oncogene, KRAS) (-), (anaplastic lymphoma kinase, ALK) (-), (ROS protooncogene 1, ROS1) (-).

The latest time for reexamination was at Aug. 10 2019. 


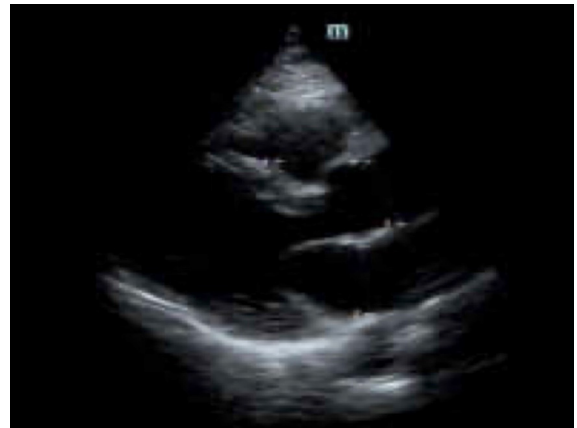

Echocardiographic examination of the heart:

$\begin{array}{llll}\text { LA } & 24 \mathrm{~mm} & \text { AO } & 24 \mathrm{~mm} \\ \text { LV } & 42 \mathrm{~mm} & \text { IVS } & 9 \mathrm{~mm} \\ \text { RA } & 27 \mathrm{~mm} & \text { LVPW } & 8 \mathrm{~mm} \\ \text { RV } & 21 \mathrm{~mm} & \text { PA } & 22 \mathrm{~mm}\end{array}$

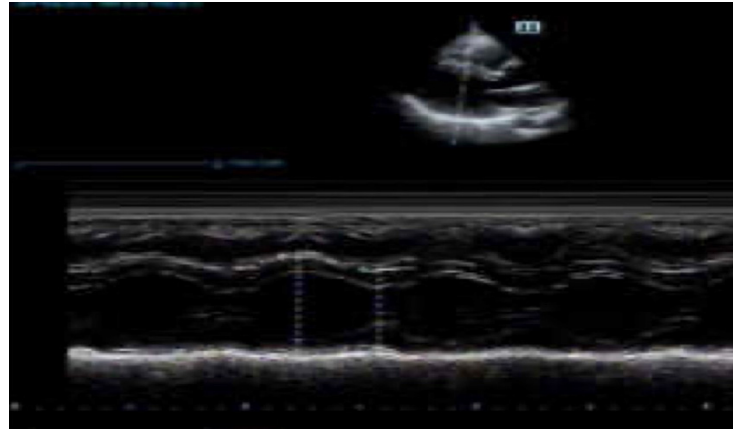

MV E $0.94 \mathrm{~m} / \mathrm{s}$

EF $77 \%$

FS $45 \%$

Figure 2 Ultrasonic cardiogram. (I) The inner diameter of the ascending aorta is normal, the wall is smooth, and the amplitude of the main wave is normal. (II) The repulse wave is present, the inner diameter of the pulmonary artery is normal, and the diameter of each atrioventricular cavity is normal. (III) The thickness of the ventricular wall and the movement are normal. The continuity of atrioventricular septum is intact, and the morphology, structure and movement of the valves are normal. (IV) There are no abnormalities in the pericardium or pericardial cavity.
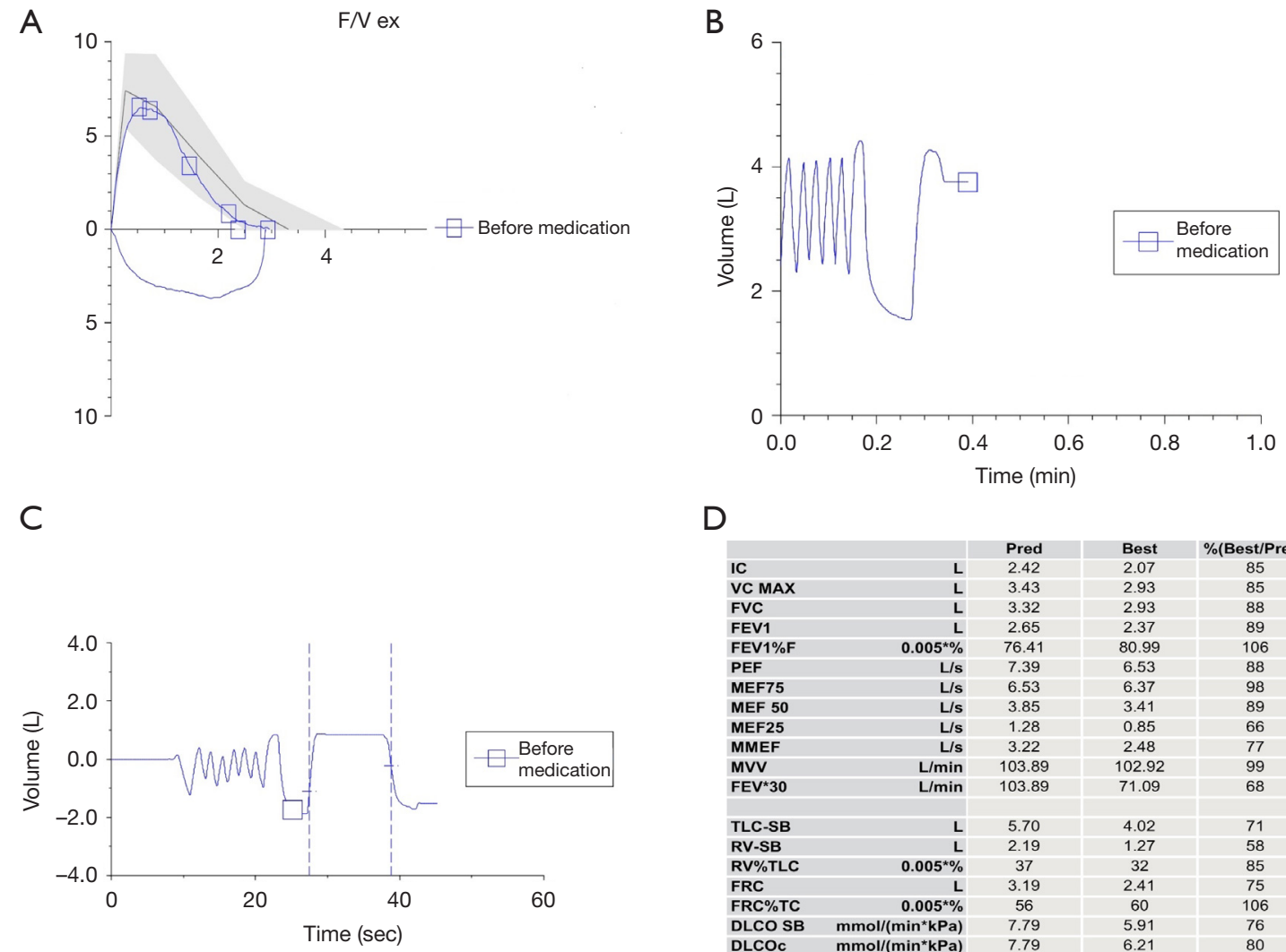

\begin{tabular}{|c|c|c|c|c|}
\hline & & Pred & Best & $\%($ Best/Pred) \\
\hline IC & L & 2.42 & 2.07 & 85 \\
\hline VC MAX & L & 3.43 & 2.93 & 85 \\
\hline FVC & L & 3.32 & 2.93 & 88 \\
\hline FEV1 & L & 2.65 & 2.37 & 89 \\
\hline FEV1\%F & $0.005^{\star \%} \%$ & 76.41 & 80.99 & 106 \\
\hline PEF & U/s & 7.39 & 6.53 & 88 \\
\hline MEF75 & $\mathrm{L} / \mathrm{s}$ & 6.53 & 6.37 & 98 \\
\hline MEF 50 & L/s & 3.85 & 3.41 & 89 \\
\hline MEF25 & L/s & 1.28 & 0.85 & 66 \\
\hline MMEF & L/s & 3.22 & 2.48 & 77 \\
\hline MVV & $\mathrm{L} / \mathrm{min}$ & 103.89 & 102.92 & 99 \\
\hline FEV $* 30$ & L/min & 103.89 & 71.09 & 68 \\
\hline TLC-SB & L & 5.70 & 4.02 & 71 \\
\hline RV-SB & L & 2.19 & 1.27 & 58 \\
\hline RV\%TLC & $0.005^{*} \%$ & 37 & 32 & 85 \\
\hline FRC & 0.000 & 3.19 & 2.41 & 75 \\
\hline FRC\%TC & $0.005^{*} \%$ & 56 & 60 & 106 \\
\hline DLCO SB & $\mathrm{mmol} /\left(\mathrm{min}^{*} \mathrm{kPa}\right)$ & 7.79 & 5.91 & 76 \\
\hline DLCOc & $\mathrm{mmol} /\left(\mathrm{min}^{*} \mathrm{kPa}\right)$ & 7.79 & 6.21 & 80 \\
\hline $\mathrm{KCOC}$ & $\mathrm{mmol} /\left(\mathrm{min}^{*} \mathrm{kPa}^{*} \mathrm{~L}\right)$ & 1.37 & 1.60 & 117 \\
\hline
\end{tabular}

Figure 3 (A) Flow volume curve. (B,C) Flow time volume. (D) Lung function index value. Pulmonary function results. (I) Lung ventilation function is normal. (II) Dispersion and residual function is normal. 


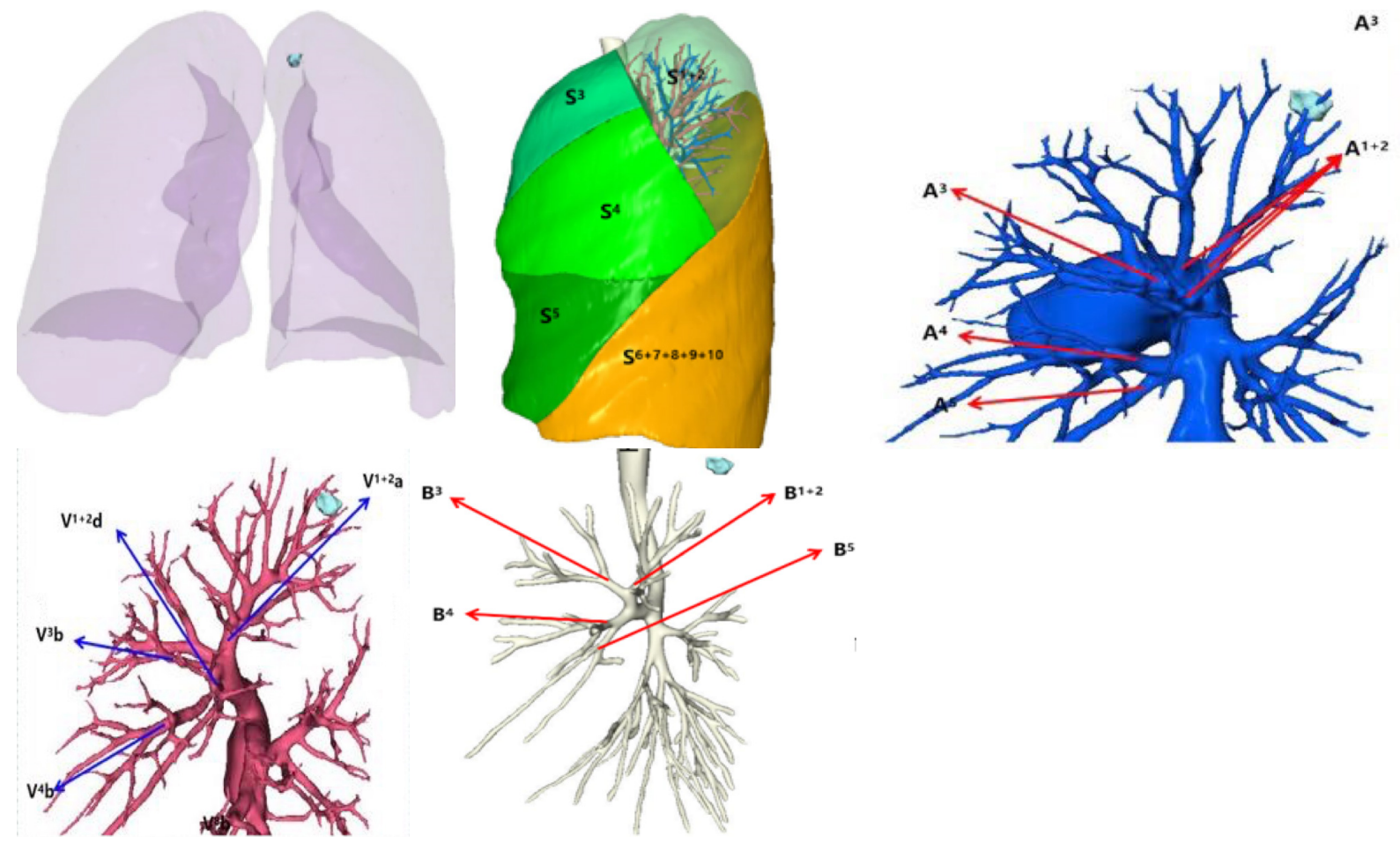

Figure 4 Preoperative 3D reconstruction results.

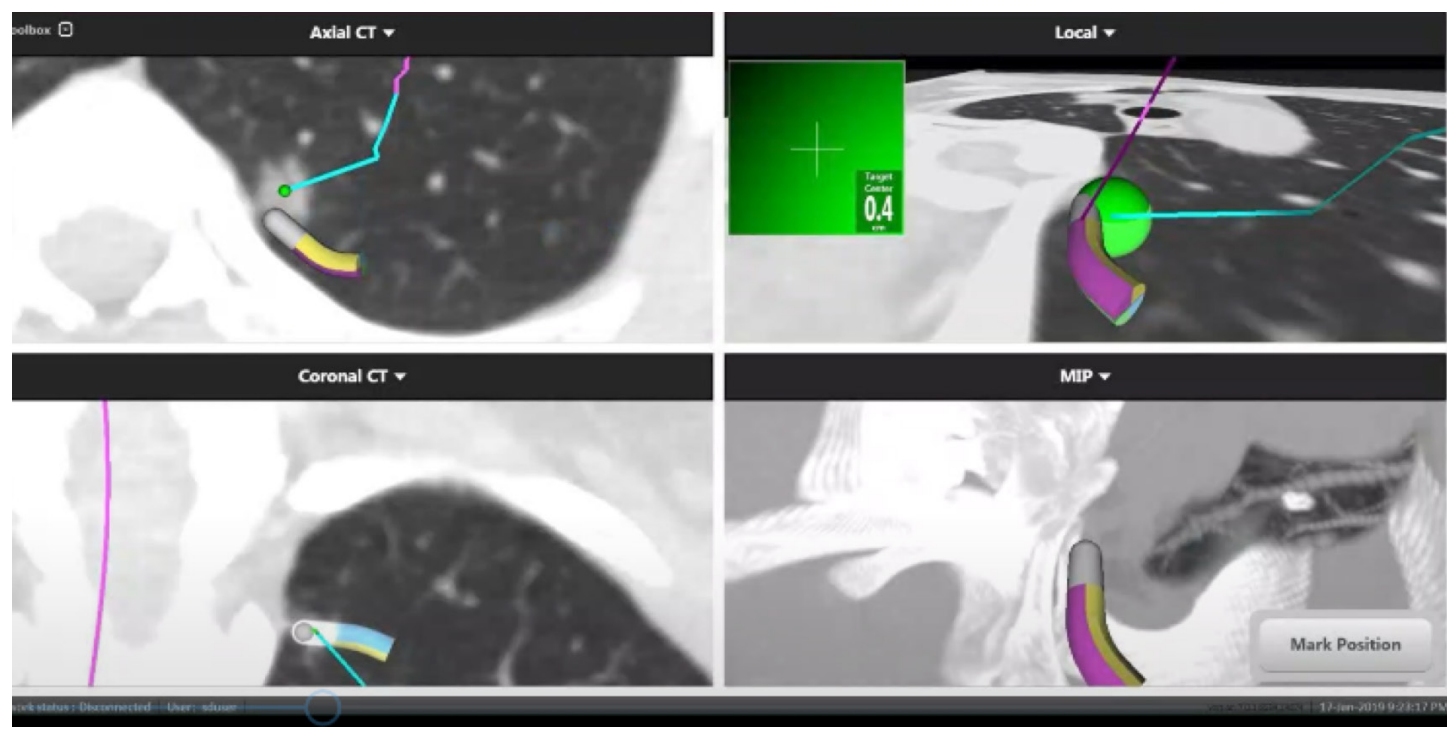

Figure 5 (I) During the operation, the sensor probe was loctated $1.0 \mathrm{~cm}$ from target lesion. Indocyanine green (ICG) was injected into the pulmonary nodules under the guidance of electromagnetic navigation. (II) The ICG solution was injected with $0.1 \mathrm{~mL}$ under the guidance of magnetic navigation (medtronic). 




Figure 6 After entering the chest cavity, the fluorescence staining area of the posterior segment of the left upper pulmonary apex was observed. The pulmonary parenchyma with the target lesion was stained with ICG before VATS resection. After the artery, vein, and trachea of the posterior segment of the left upper pulmonary apex were severed, indocyanine green was injected into the vein.
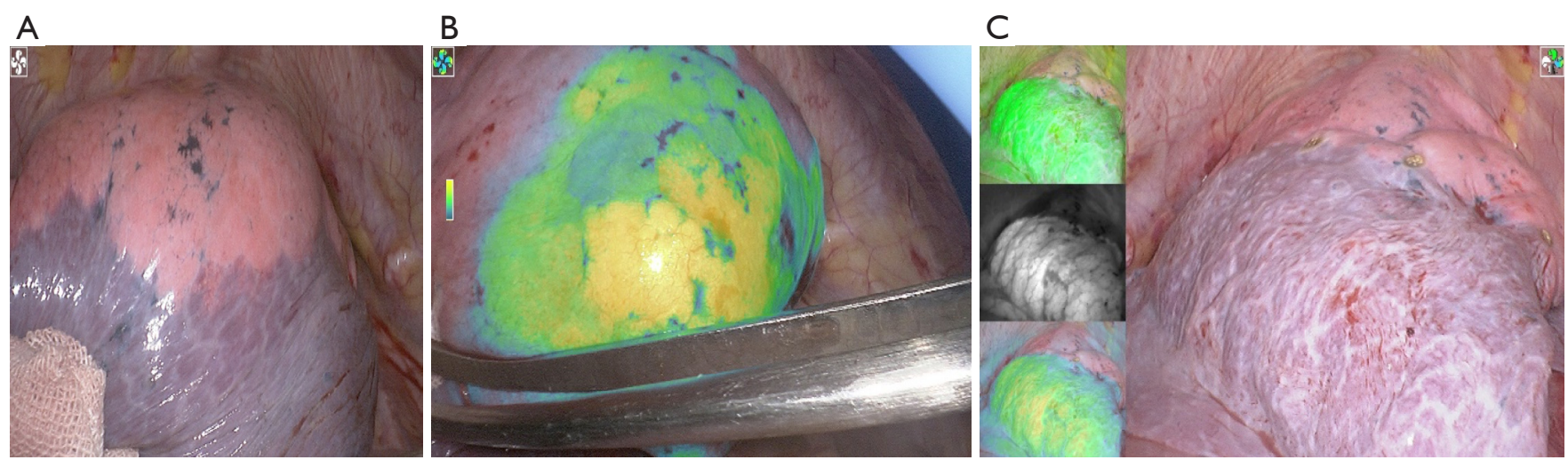

Figure 7 This is a planar view of the lung segment under a fluorescent endoscope. (A) Lung segment boundary plane in normal mode; (B) lung boundary plane in fluorescence mode; (C) three views of the lung segment.

Reexamination showed that no abnormal tumor markers were found in the lung cancer, no tumor recurrence was found in the chest and abdomen CT, and no brain metastasis was found in brain MRI.

\section{iMDT discussion}

The patient's physical examination showed that the size of the left upper lung was $9 \mathrm{~mm} \times 7 \mathrm{~mm}$. After antiinflammatory treatment for 2 weeks, no nodule regression was found in the reexamination after 3 months. Combined with the patient's clinical imaging results, the patient was highly suspected of left upper lung cancer, and no other surgical contraindications were found before the operation. The localization of the left upper lung nodule under the guidance of electromagnetic navigation and the resection of the left upper apex and posterior segment of the left upper lung assisted by electric vision were carried out. The postoperative pathology was confirmed as left upper lung adenocarcinoma in situ of the lung, with the gene test (-).

\section{Department of Radiology}

The detection of pulmonary nodules mainly depends on physical examination and screening. In the past, the means of lung cancer screening mainly depended on chest $\mathrm{X}$-ray. Due to the small size and low density of ground glass nodules, chest $\mathrm{X}$-rays easily missed many positive diagnoses. In recent years, with the widespread use of chest CT, the diagnosis of pulmonary nodules has been greatly improved. Data from the American National Lung Screening Trial showed that screening individuals with high-risk factors using low-dose CT decreased the 
mortality rate from lung cancer by $20 \%$ (3). Our patient was diagnosed with a left upper pulmonary nodule, edge burr on CT image, pleural traction in the local area, a little solid component in the mediastinal window, and was highly suspected for lung cancer. In addition, due to the diagnosis of lung ground glass nodule, it was deemed unnecessary to do positron emission tomography-CT (PET-CT). Some studies have shown that if the SUV value is more than 2.5 , it can be diagnosed as a malignant pulmonary nodule. The sensitivity and specificity is $92 \%$ and $90 \%$ respectively (4). However, for ground glass nodules (GGNs), PET-CT easily makes false negatives because of the nodule's inactive metabolism. It is suggested that PET/CT not be the first choice in the diagnosis of GGNs. Also, GGNs are usually not accompanied by lymph node metastasis and distant metastasis, which also reduces the sensitivity of PET/CT in the diagnosis of malignant lesions.

\section{Department of Thoracic Surgery}

With the popularization of low-dose spiral CT, patients with small pulmonary nodules are more frequently being found. According to the density of the nodules, they can be divided into three types: GGNS, partial solid nodules, and solid nodules. GGNs are mainly characterized by ground glass shadow, with no or a small amount of solid components. GGNs can be benign lesions, such as focal interstitial fibrosis, infection, hemorrhage, edema, etc.; they can also be atypical adenomatous hyperplasia of precancerous lesions; or malignant lesions, such as carcinoma in situ, microinvasive adenocarcinoma, invasive adenocarcinoma, etc. (5). Such nodules bring many challenges to surgeons in surgical resection. According to the American College of Chest Physicians evidence-based clinical practice guidelines, Surgical resection remains the primary approach to the treatment of stage I and II nonsmall cell lung cancer (NSCLC). Lobectomy or greater resection remains the preferred approach to $\mathrm{T} 1 \mathrm{~b}$ and larger tumors (6). The National Comprehensive Cancer Network (NCCN) guidelines also recommend lobectomy as the first choice for the treatment of early NSCLC. In recent years, more early lung cancer has been found, and segmental lung resection has also been more frequently used in surgical treatment. In addition to the patients with poor cardiopulmonary function and high risk factors or contraindications of lobectomy, NCCN guidelines indicate that segmental resection can also be performed when the nodule is less than $2 \mathrm{~cm}$. For this, 1 of the following 3 conditions should be met: (I) adenocarcinoma in situ confirmed by pathology; (II) a ground glass component of more than $50 \%$; 3 . tumor doubling time of longer than 400 days under image follow-up. In view of the $9 \mathrm{~mm} \times 7 \mathrm{~mm}$ nodule in the posterior segment of the left upper apex of the lung and a little solid component in the mediastinal window, it was difficult to find the nodule during the operation. Therefore, indocyanine green was injected into the position of the pulmonary nodule with the help of electromagnetic navigation guidance technology. During the operation, the position of the pulmonary nodule was well displayed with the help of the fluorescence endoscope. In addition, 3D reconstruction results before operation confirmed that the pulmonary nodule was located in the posterior segment of the left upper lung tip, and there was no obvious vascular variation. In view of the previous judgment of the segmental plane by the method of pulmonary expansion and atrophy, we use the method of injecting fluorescent dye through the vein to display the lung segment plane more accurately in the fluorescent mode, so as to achieve more accurate resection.

\section{Department of Internal Medicine-Oncology}

According to the eighth edition of lung cancer staging the patient was diagnosed as Tis (7). According to NCCN guidelines, adjuvant chemotherapy is not recommended for patients with stage IA disease. Postoperative chemotherapy may be considered for high-risk, margin-negative stage IB disease. Adjuvant chemotherapy is recommended for routine use in patients with stage IIA, IIB, or IIIA disease who have undergone complete surgical resections (8). The patient had no chemotherapy index after operation, and continued to have regular reexamination.

\section{Department of Radiotherapy}

At present, surgical resection is still the standard treatment for stage I or II NSCLC. Surgery provides the best chance for cure (6). There are obvious surgical contraindications for preoperative examination. For example, patients with severe cardiopulmonary disease or patients with old age, stereotactic body radiation therapy (SBRT) can be a good treatment (9). In 2015, Chang et al. showed that the 3-year OS of a SBRT group was significantly higher than that of an operation group, and there was no significant difference in the 3-year recurrence rate of lymph nodes, locoregional recurrence-free survival (LRFS) rate and distant metastasis- 
free survival (DMFS) rate. For operable early lung cancer, it can be preliminarily concluded that SBRT is not inferior to surgery (8). Compared with surgery, SBRT can be noninvasive, have no postoperative complications or loss of lung function, and can handle the characteristics of multiple lesions. For this patient, the preoperative examination was normal and thus the patient could receive more benefit from the operation. Due to the postoperative pathology is carcinoma in situ, the postoperative pathological diagnosis of early lung cancer ruled out the need for postoperative radiotherapy.

Several questions regarding the new method were further discussed as follows:

\section{Question 1. What are the international methods for nodule localization?}

\section{Expert opinion 1: Dr. Samy Lachkar \& Dr. Jean Marc Baste}

The most described method for nodule localization is the CT guided technique with blue dye marking, ICG, coils, hook, etc. However, as we emphasize in our publications $(10-12)$, the problematic is mainly logistic, involving pain and pneumothorax; that is why we propose an alternative method of pleural dye marking using Radial Endobronchial Ultrasound and Virtual Bronchoscopy before performing Sublobar Pulmonary Resection.

\section{Expert opinion 2: Dr. Naofumi Shinagawa}

There are many methods for diagnosing GGNs. Bronchoscopy with a biopsy is one option. Usually, X-ray fluoroscopy is used to localize the lesion. However, it is difficult to recognize GGNs by X-ray fluoroscopy. Radial endobronchial ultrasound is also helpful for locating GGNs in these cases. CT fluoroscopy also aids in locating the nodules for transthoracic needle aspiration. The most standardized method to diagnose GGNs is surgical resection. However, it is difficult to confirm the location of the lesion during surgery. Placing a hook wire is the most common way to locate a GGN during surgery. This technique can also cause complications such as pneumothorax and hemoptysis. Dye marking using bronchoscopy with a 3-D map of the lung or a navigation system is another option. I think dye marking using bronchoscopy is safe and very promising.

\section{Expert opinion 3: Dr. Calvin S. H. Ng}

Palpation whenever possible remains the most reliable approach for localization. For difficult to palpate nodules, localization can be divided into non-interventional or interventional. Non-interventional methods include the use of advanced 3-D CT reconstruction software to plan for procedures such as segmentectomy and estimate margins based on the location of the nodule, the segmental vessels and bronchus, and the anticipated intersegmental line. Intra-operative ultrasound in select patients could also rapidly provide the location of the nodule. As for the interventional methods, the most popular would be percutaneous hookwire localization, followed by other methods of percutaneous marking, for example using metal, radionuclide or dye marking. More recently, the use of endobronchial metal fiducial and dye marking guided by navigation bronchoscopy has gained increasing recognition.

\section{Expert opinion 4: Dr. Masaaki Sato}

There are multiple methods available including CTguided markings (hookwire, dye injection, others) and bronchoscopic methods (dye injection, microcoil, others).

\section{Expert opinion 5: Dr. Min P Kim}

There are several methods of localizing suspicious lung nodules during minimally invasive lung resection. Two broad categories are transbronchial localization and transthoracic localization. Transbronchial localization typically uses electromagnetic navigation to guide a marker to the target during the operation. Transthoracic localization can occur before the operation or during the operation. Standard transthoracic localization before surgery is performed by a radiologist who places a marker before surgery using CT. There are two techniques for transthoracic localization during the operation. The first technique is the use of cone-beam CT in the operating room. The cone-beam CT would allow for accurate placement of the marker in the operating room. The second technique is to use electromagnetic navigation transthoracic needle localization and placement of the marker. The latter technique takes significantly less time compared to the cone-beam CT technique. Each of these methods has a trade-off of risks for the patient, accuracy of the localization, and length of time in the operating room for localization procedure. The typical markers are methylene blue dye, gold fiducial, indocyanine green fluorescence, or radioisotope for all of the techniques with the addition of the use of hook wire for transthoracic localization. Each of the markers has different properties with its advantages and disadvantages and complication 
rates.

\section{Question 2. What is the prospect of magnetic navigation for nodule localization?}

Expert opinion 1: Dr. Samy Lachkar \& Dr. Jean Marc Baste

Despite its relatively good results, this technique presents some limitations, the first of which is that localization is not performed in real time, the second concerns the use of a therapeutic bronchoscope, which in turn results in less selective catheterization of the subsegmental bronchus, and finally specific OR preparation that ENB requires, fluoroscopy and expensive disposable material.

\section{Expert opinion 2: Dr. Naofumi Shinagawa}

The superDimension ${ }^{\mathrm{TM}}$ is the world's most widely used bronchoscope navigation system. This system supports the placement of fiducial markers for stereotactic irradiation and dye marking for surgical resection. It has already been widely used in North America, and its usefulness has been reported in previous studies. In a high-volume center, the use of this technique has limitations such as a complicated setting and high cost for disposable equipment. However, despite the limitations, I think that it can be considered a highly promising method.

\section{Expert opinion 3: Dr. Calvin S. H. Ng}

As in many of these navigation bronchoscopy platforms, they suffer from slight inaccuracies due to CT-body divergence. Newer platforms have improved navigation accuracy by local re-registration using fluoroscopy, or having direct endoscopic visualization to compliment the virtual navigation. Another problem with the current workflow of electromagnetic navigation marking for nodule localization is that there is no way to confirm or assess precisely the position of the marking in relation to the nodule. Cone-beam CT can provide such information, but it is both expensive and inaccessible to most institutes. Mobile CT scanners or $\mathrm{C}$-arm fluoroscopic imaging with 3-D image reconstruction could provide this essential information in the future.

\section{Expert opinion 4: Dr. Masaaki Sato}

ENB is one of the useful methods available for bronchoscopic localization. Limitations include the high cost and less accurate navigation in peripheral areas of the lung. To complement the inaccuracy, they have recently introduced a new system using cone-beam CT; however, if cone-beam CT is used anyways, what's the point of using ENB at all... that is the question. Anyways I think ENB is one of the strategies to be pursued as one of the bronchoscopic localization techniques.

\section{Expert opinion 5: Dr. Min P Kim}

Electromagnetic navigation lung nodule localization can be used in transbronchial localization as well as transthoracic localization. As articulated in this article, the transbronchial localization provides accurate localization of suspicious lung nodules that would be difficult to localize during standard minimally invasive pulmonary resection with a visual inspection. The transthoracic electromagnetic localization provides the same accuracy, but it saves a significant amount of time in the operating room compared to the transbronchial localization. In order to perform transbronchial localization, the patient is typically in the supine position with a single lumen tube intubation. The localization procedure is performed, then the patient undergoes tube exchange to the double-lumen tube and turned on the lateral decubitus position. On the other hand, the transthoracic electromagnetic localization is performed with the patient with a double-lumen tube in lateral decubitus position, which allows quicker localization without significant time spent moving patients or exchanging the endotracheal tube.

\section{Question 3. What are the application prospects for} fluorescence endoscopy in lung cancer surgery apart from the determination of the plane between segments of lung resection?

\section{Expert opinion 1: Dr. Samy Lachkar \& Dr. Jean Marc Baste}

We believe that in the future, bronchoscopy procedures should be able to target the nodule or the margin of segmentectomy, easily, precisely and during the same surgical procedure by using new smart dye and fluorescence. The use of indocyanine green (ICG) could be promising (10). ICG has been used as a contrast agent for the intraoperative detection of diverse tumor types; this is based on two characteristics of ICG: first, ICG and protein complex may passively accumulate in solid tumors due to increased vasculature and dysfunctional lymphatics [known as the enhanced permeability and retention effect (EPR)], second, ICG fluoresces at $800 \mathrm{~nm}$, and is the only near infrared (NIR) contrast agent approved 
by the Food and Drug Administration (FDA) and the European Medicines Agency (EMA) for a small number of indications in surgery. NIR fluorescence imaging is a promising technology, which can aid in intraoperative imaging. NIR light is non-invasive and non-radioactive. Moreover, it has relatively deeper tissue penetration, lower autofluorescence, and lower scattering. These advantages make NIR fluorescence imaging suitable for intraoperative imaging. Some bronchoscopy case series have shown the efficiency of this marking (11) but more studies will be needed in the future. Moreover, other fluorescent dyes have been used in clinical trials to target patients with thoracic malignancies (12).

Two others potentials advantage: per-operative fluorescence can increase the margin during wedge resection for ground glass opacity and could be a tool to guide lymphadenectomy to propose guided biopsy rather than radical lymphadenectomy.

\section{Expert opinion 2: Dr. Naofumi Shinagawa}

This question is very difficult for pulmonologists. Apart from determination of the plane, I think fluorescence endoscopy can be used to diagnose sentinel lymph node for selective sentinel lymph node dissection.

\section{Expert opinion 3: Dr. Calvin S. H. Ng}

Current use of fluorescent endoscopy in the form of indocyanine green in lung cancer surgery include determination of intersegmental plane, nodule localization, sentinel lymph node identification and lymphatic channel mapping. Future availability of tissue specific fluorescent dye could allow detection of unsuspecting nodules or metastases, and highlight of anatomical structure for easier and safer surgery.

\section{Expert opinion 4: Dr. Masaaki Sato}

First, there are multiple reports discussing about ICGmediated localization. A conventional method is to localize a pulmonary nodule after CT-mediated percutaneous injection of ICG. More recently, bronchoscopic localization has been utilized worldwide, for example, by using electromagnetic navigation bronchoscopy as the authors demonstrated. Virtual-assisted lung mapping or VAL-MAP is another strategy using bronchoscopic injection of ICG not only to localize a nodule but to indicate an appropriate resection lines by drawing a "map" with multiple dye marks on the lung. This strategy is particularly useful for complex or extended segmentectomy beyond conventional segments.

\section{Expert opinion 5: Dr. Min P Kim}

The indocyanine green fluorescence has been used effectively in minimally invasive segmentectomy. The indocyanine green fluorescence is injected into the vein after the segmental pulmonary artery is divided, and it demarks the plain between the perfused and non-perfused areas in the lung that correlates to the segmental anatomy. Additional use of the indocyanine green is its use as a marker during lung nodule localization. It provides localization during minimally invasive pulmonary nodule resection. This has been a very useful marker during robot-assisted pulmonary resection since the robot camera can easily visualize the indocyanine green in the tissue.

\section{Conclusions}

The features of ground glass nodules are partial solid or few solid components. These nodules pose significant challenges to thoracic surgeons in their operation. With the help of magnetic navigation technology before operation, the accurate positioning of such nodules, along with their accurate surgical resection, can be realized, and thus this technology can be of great assistance to young doctors. In the early stage of lung ground glass nodules, most are atypical hyperplasia, carcinoma in situ, or micro invasive cancer, with few lymph node metastases. For such patients, segmental resection can be performed after evaluation. With 3D reconstruction technology before operation, accurate segmentation of the lung nodule can be performed to understand the blood vessels around the nodule and whether there is variation, after which segmental resection can be performed. In order to effectively preserve the lung function and improve the survival treatment of patients, the expansion and atrophy method has been primarily used to identify the lung segmental plane. Such methods require a 20-30-minute wait during the operation. After the disconnection of the vein, artery and trachea, intravenous injection of indocyanine green can be used to identify the lung segmental plane more quickly and accurately, shorten the operation time, and is thus more in line with the concept of rapid rehabilitation. Overall, for early non-small cell lung cancer, there is no drawback to performing a preoperative examination, and surgery is the preferred treatment. When combined with other auxiliary technologies, surgery is more accurate, less traumatic, and provides faster recovery and greater benefit to patients. 


\section{Acknowledgments}

Funding: None.

\section{Footnote}

Reporting Checklist: The authors have completed the CARE reporting checklist. Available at http://dx.doi.org/10.21037/ jtd-20-2089

Conflicts of Interest: All authors have completed the ICMJE uniform disclosure form (available at http://dx.doi. org/10.21037/jtd-20-2089). CSN reports Consultant \& Advisory Board for Siemens Healthineer; Consultant \& Advisory Board for Medtronic; Consultant \& Advisory Board for Johnson \& Johnson; Consultant \& Advisory Board for Stryker. CS Ng also serves as an unpaid editorial board member of Journal of Thoracic Disease from Feb 2019 to Jan 2021. MPK reports personal fees from Veran, personal fees from Medtronics, personal fees from Intuitive Surgical, outside the submitted work. MP Kim also serves as an unpaid editorial board member of Journal of Thoracic Disease from Sep 2018 - Aug 2020. SL reports personal fees from OLYMPUS, personal fees from FUJIFILM, personal fees from BOSTON Scientific, personal fees from Ambu, outside the submitted work. $M$ Sato serves as an unpaid editorial board member of Journal of Thoracic Disease from Oct 2018 to Sep 2020.

Ethical Statement: The authors are responsible for all aspects of the work and to ensure that issues related to the accuracy or completeness of any part of the work are properly investigated and resolved. The case report and related pictures were published with the informed consent of the patient and all procedures performed in studies involving human participants were in accordance with the ethical standards of the institutional and/or national research committee(s) and with the Helsinki Declaration (as revised in 2013). Written informed consent was obtained from the patient.

Open Access Statement: This is an Open Access article distributed in accordance with the Creative Commons Attribution-NonCommercial-NoDerivs 4.0 International License (CC BY-NC-ND 4.0), which permits the noncommercial replication and distribution of the article with the strict proviso that no changes or edits are made and the original work is properly cited (including links to both the formal publication through the relevant DOI and the license). See: https://creativecommons.org/licenses/by-nc-nd/4.0/.

\section{References}

1. Bray F, Ferlay J, Soerjomataram I, et al. Global cancer statistics 2018: GLOBOCAN estimates of incidence and mortality worldwide for 36 cancers in 185 countries [published correction appears in CA Cancer J Clin. 2020 Jul;70(4):313]. CA Cancer J Clin 2018;68:394-424.

2. Chen $W$, Zheng R, Baade PD, et al. Cancer statistics in China, 2015. CA Cancer J Clin 2016;66:115-32.

3. National Lung Screening Trial Research Team, Aberle DR, Adams AM, et al. Reduced lung-cancer mortality with low-dose computed tomographic screening. N Engl J Med 2011;365:395-409.

4. Lowe VJ, Fletcher JW, Gobar L, et al. Prospective investigation of positron emission tomography in lung nodules. J Clin Oncol 1998;16:1075-84.

5. Park CM, Goo JM, Lee HJ, et al. Nodular groundglass opacity at thin-section CT: histologic correlation and evaluation of change at follow-up. Radiographics 2007;27:391-408.

6. Howington JA, Blum MG, Chang AC, et al. Treatment of stage I and II non-small cell lung cancer: Diagnosis and management of lung cancer, 3rd ed: American College of Chest Physicians evidence-based clinical practice guidelines. Chest 2013;143:e278S-e313S.

7. Goldstraw P, Chansky K, Crowley J, et al. The IASLC Lung Cancer Staging Project: Proposals for Revision of the TNM Stage Groupings in the Forthcoming (Eighth) Edition of the TNM Classification for Lung Cancer. J Thorac Oncol 2016;11:39-51.

8. Kris MG, Gaspar LE, Chaft JE, et al. Adjuvant Systemic Therapy and Adjuvant Radiation Therapy for Stage I to IIIA Completely Resected Non-Small-Cell Lung Cancers: American Society of Clinical Oncology/Cancer Care Ontario Clinical Practice Guideline Update. J Clin Oncol 2017;35:2960-74.

9. Donington J, Ferguson M, Mazzone P, et al. American College of Chest Physicians and Society of Thoracic Surgeons consensus statement for evaluation and management for high-risk patients with stage I non-small cell lung cancer. Chest 2012;142:1620-35.

10. Lachkar S, Baste JM, Salaün M, et al. All things are created twice, but the surgeon only gets one chance: bronchoscopy marking may help the surgeon to perform sublobar resection. J Thorac Dis 2018;10:E758-60. 
11. Baste JM, Soldea V, Lachkar S, et al. Development of a precision multimodal surgical navigation system for lung robotic segmentectomy. J Thorac Dis 2018;10:S1195-S1204.

12. Lachkar S, Baste JM, Thiberville L, et al. Pleural Dye
Marking Using Radial Endobronchial Ultrasound and Virtual Bronchoscopy before Sublobar Pulmonary Resection for Small Peripheral Nodules. Respiration 2018;95:354-61.

Cite this article as: Wang G, Lin Y, Zheng L, Liang Y, Zhao L, Wen Y, Zhang R, Huang Z, Yang L, Zhao D, Lachkar S, Baste JM, Shinagawa N, Ng CSH, Sato M, Kim MP, Zhang L. A new method for accurately localizing and resecting pulmonary nodules. J Thorac Dis 2020;12(9):4973-4984. doi: 10.21037/jtd-202089 